\title{
Review
}

\section{Muscular Dystrophy with Ribitol- Phosphate Deficiency: A Novel Post-Translational Mechanism in Dystroglycanopathy}

\author{
Motoi Kanagawa ${ }^{\mathrm{a}, *}$ and Tatsushi Toda $\mathrm{a}^{\mathrm{a}, \mathrm{b}, *}$ \\ ${ }^{a}$ Division of Neurology/Molecular Brain Science, Kobe University Graduate School of Medicine, \\ Chuo-ku, Kobe, Japan \\ ${ }^{\mathrm{b}}$ Department of Neurology, Division of Neuroscience, Graduate School of Medicine, \\ The University of Tokyo, Bunkyo-ku, Tokyo, Japan
}

\begin{abstract}
Muscular dystrophy is a group of genetic disorders characterized by progressive muscle weakness. In the early 2000s, a new classification of muscular dystrophy, dystroglycanopathy, was established. Dystroglycanopathy often associates with abnormalities in the central nervous system. Currently, at least eighteen genes have been identified that are responsible for dystroglycanopathy, and despite its genetic heterogeneity, its common biochemical feature is abnormal glycosylation of alpha-dystroglycan. Abnormal glycosylation of alpha-dystroglycan reduces its binding activities to ligand proteins, including laminins. In just the last few years, remarkable progress has been made in determining the sugar chain structures and gene functions associated with dystroglycanopathy. The normal sugar chain contains tandem structures of ribitol-phosphate, a pentose alcohol that was previously unknown in humans. The dystroglycanopathy genes fukutin, fukutin-related protein (FKRP), and isoprenoid synthase domain-containing protein (ISPD) encode essential enzymes for the synthesis of this structure: fukutin and FKRP transfer ribitol-phosphate onto sugar chains of alpha-dystroglycan, and ISPD synthesizes CDPribitol, a donor substrate for fukutin and FKRP. These findings resolved long-standing questions and established a disease subgroup that is ribitol-phosphate deficient, which describes a large population of dystroglycanopathy patients. Here, we review the history of dystroglycanopathy, the properties of the sugar chain structure of alpha-dystroglycan, dystroglycanopathy gene functions, and therapeutic strategies.
\end{abstract}

Keywords: Muscular dystrophy, dystroglycanopathy, dystroglycan, glycosylation, ribitol-phosphate, fukutin, fukutin-related protein, isoprenoid synthase domain containing protein

\footnotetext{
${ }^{*}$ Correspondence to: Motoi Kanagawa, Division of Neurology/Molecular Brain Science, Kobe University Graduate School of Medicine, 7-5-1 Kusunoki-cho, Chuo-ku, Kobe 650-0017, Japan. Tel.: +81 78382 6287; E-mail: kanagawa@med.kobe-u.ac.jp and Tatsushi Toda, Department of Neurology, Division of Neuroscience, Graduate School of Medicine, The University of Tokyo, 7-3-1 Hongo, Bunkyo-ku, Tokyo 113-8655, Japan. Tel.: +81 35800 6542; E-mail: toda@m.u-tokyo.ac.jp.
}

\section{HISTORY OF GLYCOSYLATION- DEFICIENT MUSCULAR DYSTROPHIES}

Muscular dystrophies are a heterogeneous group of genetic disorders characterized by the progressive loss of muscle strength and integrity, and are categorized into subgroups based on factors such as the responsible genes, inheritance patterns and clinical presentation. Currently, more than 40 genes have 
been identified whose mutations cause different types of muscular dystrophies (www.musclegenetable.fr). The most common type is Duchenne-type muscular dystrophy (DMD), and the gene responsible for DMD, dystrophin, was identified in 1987 [1]. Dystrophin encodes a large actin-binding cytoskeletal protein located underneath the muscle plasma membrane. After the discovery of dystrophin, several transmembrane glycoproteins that co-purified with dystrophin protein were identified [2]. These proteins form a large protein complex that is called the dystrophin-glycoprotein complex (DGC). The components of the DGC include sarcoglycans (SGs), sarcospan, and dystroglycan (DG) [3]. The major function of the DGC is connecting the basement membrane to dystrophin-actin cytoskeleton across the plasma membrane. In addition to dystrophin, several components of the DGC or basement membrane are associated with muscular dystrophies [4]. Mutations in SGs are associated with limb-girdle type muscular dystrophies (LGMDs), and mutations in the laminin alpha2 chain, a basement membrane protein that is a direct ligand for the DGC, are associated with congenital type muscular dystrophy 1A (MDC1A). Biochemical and pathophysiological studies have further established that the DGC serves as a physical link between the basement membrane and the cytoskeleton, which provides mechanical stability to the muscle plasma membrane.

DG, which serves as the central component of the DGC, is encoded by a single mRNA and is post-translationally cleaved into two subunits, $\alpha$ - and $\beta$-DG. $\alpha$-DG is a highly glycosylated extracellular subunit and serves as a receptor protein for laminin in the basement membrane. Glycosylation is required for ligand binding activities of $\alpha$-DG. $\beta$-DG is a transmembrane subunit and anchors $\alpha$-DG on the plasma membrane and intracellularly binds to dystrophin. As mentioned, mutations in SGs or laminin alpha 2 were identified as causes of muscular dystrophies in the 1990s, whereas no mutation in the DG gene, DAG1, was identified until 2011 [5]. However, in 2001 Arahata's group reported abnormal glycosylation of $\alpha$-DG in patients suffering from Fukuyama type congenital muscular dystrophy (FCMD) [6]. Subsequently, Campbell's group showed direct evidence for abnormal glycosylation of $\alpha$-DG with a severe reduction in ligand binding activities in patients with muscle-eye-brain disease (MEB) and Walker-Warburg syndrome (WWS) in addition to
FCMD [7]. Abnormal glycosylation can be determined by immunostainings of patients' biopsies with IIH6 antibody [8], which recognizes functionally glycosylated and ligand-binding forms of $\alpha$-DG. Because the common biochemical feature of these diseases is abnormal glycosylation of $\alpha$-DG, these conditions have been collectively called dystroglycanopathy (DGpathy) $[9,10]$. By the early 2000s, six genes were known as causative genes for DGpathy (fukutin, POMGNT1, POMT1, POMT2, fukutinrelated protein, and $L A R G E$ ) [11-17]. Now, eighteen genes are known to be associated with DGpathy, including the DG gene DAGl itself. Currently, DGpathies can be classified as primary (caused by mutations in the DG gene itself), secondary (caused by mutations in genes encoding proteins that directly modify $\alpha-D G$ ), or tertiary (caused by mutations in genes encoding proteins that indirectly modify $\alpha-D G$ but affect $\alpha$-DG function). The clinical phenotype of DGpathy shows a wide spectrum. The most severe end of the spectrum is characterized by CMD with extensive structural abnormalities in the brain and eye, which typically results in early infantile death. Patients at the mildest end of the spectrum might present in adult life with LGMD without brain or eye involvement. New Online Mendelian Inheritance in Man (OMIM) entries have created a simplified classification scheme for DGpathy (MDDG; muscular dystrophy dystroglycanopathy) by combining three broad phenotypic groups and gene defects [18]. In this classification scheme, DGpathy is divided into three groups: (A) CMD with brain/eye abnormalities, (B) CMD with milder brain structural abnormalities, and (C) LGMD. In addition, the causative gene is indicated numerically as shown in Table 1. According to this classification, for example, typical FCMD is "MDDG type A4."

\section{SUGAR CHAIN STRUCTURE AND DGPATHY GENE FUNCTIONS}

It is clear that the sugar chain on $\alpha$-DG underlies the pathogenesis of DGpathy; however, the sugar chain structure associated with ligand binding activity and DGpathy gene functions were long unrevealed. Recently, the structure of the sugar chain required was finally revealed. Here, we review the sugar chain structure and DGpathy gene functions while focusing on recent findings (Fig. 1). 
Table 1

$\alpha$-DGpathy genes, their functions, and MDDG number

\begin{tabular}{|c|c|c|}
\hline$\alpha$-DGpathy genes & Gene functions & $\begin{array}{l}\text { MDDG } \\
\text { number }\end{array}$ \\
\hline POMT1 & $O$-Man transferase (POMT1/2 complex) & 1 \\
\hline POMT2 & $O$-Man transferase (POMT1/2 complex) & 2 \\
\hline POMGNT1 & Protein $O$-Man $\beta 1,2$-GlcNAc transferase & 3 \\
\hline FKTN & RboP transferase & 4 \\
\hline FKRP & RboP transferase & 5 \\
\hline LARGE & $\alpha 3$-Xyl- and $\beta 3$-GlcA transferase & 6 \\
\hline ISPD & CDP-ribitol pyrophosphorylase (CDP-ribitol synthase) & 7 \\
\hline POMGNT2 & Protein $O$-Man $\beta 1,4$-GlcNAc transferase & 8 \\
\hline DAG1 & Dystroglycan & 9 \\
\hline TMEM5 & $\beta 1,4-X y l$ transferase & 10 \\
\hline B3GALNT2 & $\beta 1,3-$ GalNAc transferase & 11 \\
\hline POMK & $O$-Man kinase & 12 \\
\hline B4GAT1 & $\beta 1,4-G l c A$ transferase & 13 \\
\hline GMPPB & GDP-Man pyrophosphorylase B & 14 \\
\hline DPM1 & Dol-P-Man synthase & - \\
\hline DPM2 & Dol-P-Man synthase & - \\
\hline DPM3 & Dol-P-Man synthase & - \\
\hline DOLK & Dolichol kinase & - \\
\hline
\end{tabular}

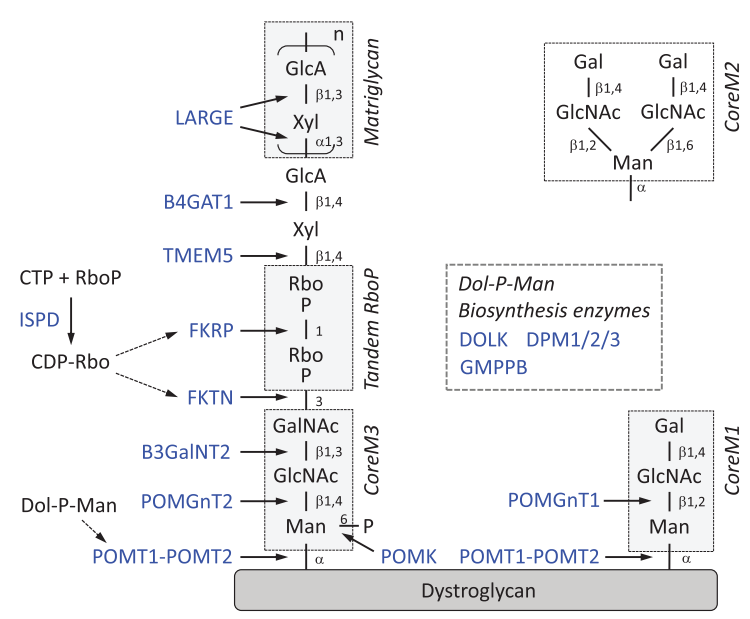

Fig. 1. Sugar chain structure of $\alpha-D G$ and functions of DGpathy genes. DGpathy gene products are written in blue and their functions are indicated by arrows. Note that nomenclature for the "Core" structure denoting $O$-Man extended only by GlcNAc residues has been also proposed [65]. RboP, ribitol 5phosphate; GlcA, glucuronic acid; Xyl, xylose; GalNAc, $\mathrm{N}$-acetylgalactosamine; GlcNAc, $\mathrm{N}$-acetylglucosamine; Man, mannose; Dol-P-Man, dolicholphosphate mannose.

\section{CoreM1 and CoreM3 glycan}

Glycosylation of proteins is characterized by the patterns of attachment of sugar moieties to proteins. For example, $N$-linked and $O$-linked glycans are attached on Asn and Ser/The residues, respectively. $\alpha$-DG possesses both $N$ - and $O$-linked glycans. In the mucin-like domain of $\alpha-\mathrm{DG}$, there is a cluster of $O$-linked glycans, and $O$-linked glycosylation is required for ligand binding activities [3]. In 1997, a unique glycan was identified, Gal- $\beta 1,4-G l c N A c-$ $\beta 1,2-\mathrm{Man}-O$ [19], and later this structure was named CoreM1 [20]. The initial sugar attached to the $\alpha$-DG core protein is mannose (Man), so such sugar chains are referred as $O$-Man types. For CoreM1 biosynthesis, the initial Man transfer to the Thr/Ser residues of $\alpha$-DG is catalyzed by a POMT1/POMT2 (protein $O$-mannosyl transferase 1/2) heterocomplex [21], and the GlcNAc- $\beta 1,2-$ Man linkage is synthesized by POMGnT1 [12]. POMTs and POMGnT1 were originally identified as causative genes for WWS and MEB, respectively [12-14]. CoreM1 itself is not directly involved in ligand binding but is thought to play a critical role in the synthesis of the ligand binding moiety that is built on other $O$-Man type glycans [22]. CoreM1 can be further modified with $\beta 1,6-$ GlcNAc branch (CoreM2) [20], but this structure accounts for a very minor population of glycans from skeletal muscle $\alpha$-DG $(<0.1 \%)$ [23].

In 2010, another type of $O$-Man glycan was identified, GalNAc- $\beta 1,3-$ GlcNAc- $\beta 1,4-M a n-O$ [24], and later named CoreM3 [20]. The initial Man transfer is also catalyzed by the POMT1/POMT2 complex, the GlcNAc $\beta 1-4$ Man linkage is synthesized by POMGnT2, and the GalNAc $\beta 1-3$ GlcNAc linkage is synthesized by B3GalNT2 [25]. The $O$-Man residue in CoreM3 is phosphorylated at the 6 position by protein $O$-Man kinase (POMK) [25]. These genes were relatively new DGpathy genes identified after 2012 [26-28]. The role of this phosphorylation is 
not known, but it is likely required for subsequent modification with the ribitol-phosphate group (see below). Overall, CoreM3 serves as a scaffold for the sugar moiety that directly interacts with ligand proteins.

\section{Matriglycan (LARGE-glycan)}

LARGE (Like-acetylglucosaminyltransferase) is the causative gene for congenital muscular dystrophy 1D (MDC1D) and the spontaneous mutant Large $e^{\text {myd }}$ (myd) mouse [17, 29]. In 2004, a report showed that the overexpression of LARGE increases the glycosylation and ligand-binding activities of $\alpha-D G$, which suggested that LARGE is involved in the biosynthesis of its ligand-binding moiety [30, 31]. In 2012, LARGE was identified as possessing two glycosyltransferase activities, $\alpha 3$-xylosyltransferase and $\beta 3$-glucuronyltransferase, which generate the disaccharide repeat [-3GlcA $\beta 1-3 \mathrm{Xyl} \alpha 1]_{\mathrm{n}}(\mathrm{GlcA}-\mathrm{Xyl}$ repeat) [32]. Enzymatically synthesized GlcA-Xyl repeats bind to laminin and are recognized by IIH6 antibody. Later, these repeating units were detected in recombinant $\alpha-D G$, which efficiently interacts with ligand proteins [33]. From these findings, the repeat structure was proven to serve as the ligand-binding moiety of $\alpha$-DG and has been named "matriglycan" [20].

Currently, known ligand proteins include laminins, agrin, perlecan, neurexin, pikachurin, and slit, all of which contain laminin G-like (LG) domains that serve as binding sites for $\alpha$-DG [34]. A recent crystal structure analysis revealed the atomic details of ligand binding. A single GlcA-Xyl disaccharide straddles $\mathrm{Ca}^{2+}$ ion in the LG4 domain of the laminin $\alpha 2$-chain [35]. This chelating binding mode explains the high affinity of this protein-carbohydrate interaction. Multiple GlcA-Xyl repeats are thought to increase the apparent affinity for the LG domains present in ligand proteins.

For the matriglycan modification, in addition to the LARGE enzyme activities, physical proteinprotein interactions between LARGE and the $\alpha$-DG $\mathrm{N}$-terminal domain are required [31]. During the process of $\alpha$-DG maturation, the $\mathrm{N}$-terminal domain functions as a recognition target by LARGE, which may ensure the LARGE-dependent modification of GlcA-Xyl repeats. In 2011, a mutation in the $\mathrm{N}$-terminal domain of $\alpha$-DG was identified in a patient with LGMD with cognitive impairment; the mutation impairs the LARGE-DG interaction and consequently reduces matriglycan modification [5].
This was the first case in which a mutation in the DG gene (DAGl) itself was known to cause muscular dystrophy ("primary" DGpathy).

\section{Tandem ribitol-phosphate}

In 2012, mutations in the ISPD gene, which encodes isoprenoid synthase domain containing protein, were identified to be the second most common cause of WWS [36, 37]. Later, a growing number of DGpathy patients who carry mutations in $I S P D$ were reported [38]. The ISPD protein belongs to the family of 4-diphosphocytidyl-2C-methylD-erythritol (CDP-ME) synthases, also known as 2-C-methyl-D-erythritol 4-phosphate (MEP) cytidylyltransferases. In bacteria, IspD functions as part of the MEP pathway of isoprenoid synthesis, but this pathway is not present in humans. Therefore, the functional role of human ISPD in $\alpha$-DG glycosylation and DGpathy remained to be elucidated. In 2015, a crystal structural analysis revealed that human ISPD cytidyltransferase domain superimposes well with a series of closely related prokaryotic cytidyltransferases and ISPD homologs including bacterial TarI [39]. In fact, the data demonstrated that human ISPD possesses cytidyltransferase activities toward pentose phosphates, suggesting that human ISPD may be a cytidyltransferase that produces novel nucleotide sugars essential for functional glycosylation of $\alpha-\mathrm{DG}$; however, the relationship between this and the sugar chain structure was still missing. Shortly thereafter, three groups independently confirmed that human ISPD catalyzes CDP-ribitol synthesis as CDPribitol pyrophosphorylase [33, 40, 41]. Meanwhile, a Japanese group found a novel moiety that contains tandemly connected ribitol-phosphates (RboP) between matriglycan and CoreM3 by mass spectrometry [33]. Ribitol is a sugar alcohol that was not known to be used in humans. In bacteria, RboP is used as a component of the teichoic acids present in cell walls, and the RboP polymer is synthesized by enzymes that use CDP-Rbo as a donor substrate. In bacteria, CDP-Rbo is synthesized by the enzyme TarI from cytidine triphosphate (CTP) and RboP. These studies indicated that human ISPD provides CDP-Rbo, which is used to synthesize the tandem RboP structure in the sugar chain of $\alpha$-DG.

The next question to answer was what gene products catalyze the synthesis of the tandem RboP structure. The fukutin family was predicted to encode a phosphoryl-ligand transferase [42, 43]. Fukutinrelated protein $(F K R P)$ was originally identified 
based on its sequence homology with fukutin [15]. Both fukutin and FKRP proteins contain the putative catalytic DXD motif, which is a conserved motif found in many families of glycosyltransferases. Therefore, both fukutin and FKRP were primary candidates for the tandem RboP biosynthesis. Indeed, the Japanese group revealed that fukutin transfers RboP from a CDP-Rbo to GalNAc in CoreM3, and then FKRP transfers RboP from a CDP-Rbo to the first RboP [33]. Thus, the tandem RboP structure was shown to be synthesized through the sequential enzymatic actions of fukutin and FKRP as RboP transferases. Several lines of evidence have also shown that there is a GlcA $\beta 1-4 \mathrm{Xyl}$ unit, which is formed independently of LARGE, between matriglycan and the tandem RboP. This structure serves as a primer for the initiation of matriglycan formation, and is synthesized by B4GAT1, which exhibits $\beta 4$-glucuronyltransferase activity, and by TMEM5, which exhibits Xyl transferase activity to form a Xylß1-4Rbo5P linkage [44-48]. It has been proposed that TMEM5 could be renamed RXYLT1 on the basis of its function (Rbo5P $\beta 1,4-X y l$ transferase) $[49,50]$.

A recent review article refers to TMEM5 as a ribitol $\beta 1,2$-xylosyltransferase [49], which differs from the nomenclature in the original report by Manya et al (ribitol $\beta 1,4-x y l o s y l t r a n s f e r a s e)$ [47]. This difference is based on whether CDP-ribitol is recognized by the researcher as an organic compound (R/S nomenclature) or a sugar derivative (D/L nomenclature). The IUPAC name of CDP-Rbo is [[(2R,3S,4R,5R)-5-(4amino-2-oxopyrimidin-1-yl) -3,4-dihydroxyoxolan2-yl] methoxy-hydroxyphosphoryl] [(2R,3S,4S)-2,3, 4,5-tetrahydroxypentyl] hydrogen phosphate. As for ribitol-phosphate, D-ribitol-5-phosphate is the preferred name as per IUPAC nomenclature for the sugar derivative. Based on the IUPAC recommendation for nomenclature of carbohydrates that includes alditols (D/L representation), CDP-ribitol is defined as CDP-D-ribitol in which the C5 position of ribitol is modified with phosphate. According to this nomenclature, the structure of tandem RboP with a Xyl modification is Xyl $\beta 1,4-R b o 5 P-1 R b o 5 P$. However, CDP-L-ribitol is also found to be named according to $\mathrm{R} / \mathrm{S}$ nomenclature for CDP-ribitol. Using this nomenclature, the structure can be alternatively referred to as Xylß1,2-Rbo1P-5Rbo1P and TMEM5 can be referred to as ribitol $\beta 1,2-$ xylsyltransferase. In this review, we pointed out this inconsistency in nomenclature stemming from the mixed use of R/S and D/L nomenclature, and have used the nomenclatures that were originally reported in research articles.

\section{Dolichol-phosphate-Man synthesis pathway}

The other DGpathy genes are involved in the dolichol-phosphate-Man (Dol-P-Man) synthesis pathway [51-55]. Dol-P-Man serves as a donor for Man residues, and the POMT1/POMT2 complex utilizes Dol-P-Man for transferring Man to $\alpha$-DG core protein. Defects in the Dol-P-Man synthesis pathway result in reduced levels of Dol-P-Man production thus affecting $O$-mannosyl glycosylation of $\alpha$-DG. Details of the Dol-P-Man pathway are documented elsewhere [56], and here we briefly review genes known to associate with DGpathy. Dol-P-Man is synthesized from GDP-Man and Dol-P by the DPM synthase complex, which consists of the catalytic component DPM1 and the ER-localized transmembrane proteins DPM2 and DPM3. Dolichol kinase (DOLK) is responsible for the formation of Dol-P. GDP-mannose pyrophosphorylase $\mathrm{B}$ (GMPPB) is the $\beta$-subunit of the essential enzyme GDP-mannose pyrophosphorylase, which catalyzes the formation of GDP-Man from Man1-phosphate and GTP. Mutations in these genes [51-55] and in ISPD [36-38] indirectly affect $\alpha$ DG function, and thus the diseases caused by these gene mutations are categorized as tertiary DGpathies. It is notable that in addition to $O$-mannosylation, Dol-P-Man acts as a Man donor in $\mathrm{N}$-glycosylation and in glycophosphatidylinositol-anchor biosynthesis. Therefore, defects in the Dol-P-Man pathway can be associated with human diseases other than DGpathy. Congenital disorder of glycosylation (CDG) was originally defined as diseases caused by defects in the $\mathrm{N}$-glycosylation process, but now includes $O$-linked and lipid glycosylation defects. Several cases of combined deficiency of protein $N$-glycosylation and $\alpha$-DG O-mannosyl glycosylation in CDG patients with $\alpha$-DGpathy have been reported [51, 53, 54].

\section{MUSCULAR DYSTROPHY WITH RIBITOL-PHOSPHATE DEFECTS}

Recent studies have established that RboP modification is indispensable for functional maturation of $\alpha$-DG, and have determined the enzyme activities of ISPD, fukutin, and FKRP that are required for this modification. Fukutin-deficient FCMD is the predominant form of DGpathy in Japan, and FKRPdeficient LGMD2I is the most frequent form of DGpathy in the U.S. and Europe. The exact number 
of patients who are ISPD-deficient is not known, but it has been reported that ISPD mutation is the second highest cause of WWS [36, 37]. Together, diseases caused by defects in these three genes can be subgrouped as DGpathy with RboP deficiency, and this group accounts for the majority of DGpathy in the world. In the remainder of this section we will review FCMD, the first identified DGpathy, and fukutin, its responsible gene.

FCMD, which was first identified by Dr. Yukio Fukuyama, is characterized by severe CMD with central nervous system and eye abnormalities [57]. FCMD is an autosomal recessive muscular dystrophy and is the second most common childhood muscular dystrophy next to DMD in Japan. The disease incidence is $\sim 3 / 100,000$, and one person in approximately 90 is expected to be a heterozygous carrier. FCMD patients manifest muscle weakness and hypotonia by early infancy [58]. Brain malformations characterized by micropolygyria of the cerebrum and cerebellum, and type II lissencephaly as well as mental retardation are associated with FCMD [59]. Eye abnormalities include myopia, cataracts, abnormal eye movement, pale optic discs, and retinal detachment. The major mutation in FCMD is a SVA retrotransposon insertion in the $3^{\prime}$ noncoding region of fukutin, which accounts for $\sim 87 \%$ of the FCMD chromosome [11]. It is notable that FCMD is the first human disease identified to be caused by an ancient retrotransposal insertion. This insertion contains a strong splice acceptor site, which induces a rare alternative donor site in the final exon, thus leading to abnormal mRNA splicing (exon-trapping) [60]. The resulting product lacks a portion of the C-terminus and instead acquires extra amino acids derived from the transposal insertion. This aberrant fukutin product loses its function in $\alpha$-DG modification. Patients heterozygous for the SVA insertion and for other mutations, such as nonsense and missense mutations, develop more severe or milder phenotypes than typical FCMD patients depending on the deleterious effects of the non-transposal mutations.

\section{THERAPEUTIC STRATEGIES FOR RIBITOL-PHOSPHATE DEFICIENT-TYPE DGPATHY}

Several therapeutic strategies for RboP-deficient type DGpathy have been proposed, and here we introduce three of them. First, since DGpathies are single gene disorders, viral vector-mediated gene therapy is the most straightforward strategy. In fact, the effectiveness of fukutin- or FKRP-gene delivery has been proven using FCMD and LGMD2I model mice, respectively [61, 62]. Second, for FCMD, an innovative strategy based on the molecular pathomechanism has been proposed. As introduced above, the unique retrotransposal insertion causes abnormal splicing, and the introduction of antisense nucleotides that target the splice acceptor and the splicing enhancer sequences prevents the pathogenic exon trapping. This antisense treatment rescues normal fukutin mRNA expression and protein production, and consequently, $\alpha$-DG glycosylation is restored in the FCMD patients' cells and in model mice [60]. This strategy can be applied to almost all FCMD patients in Japan. Third, since ISPD deficiency leads to a loss of or severe reduction in cellular CDP-ribitol, the supplementation of CDP-ribitol may be effective. This hypothesis is supported by the addition of CDPribitol into the cell culture media of ISPD-deficient cells, which restores $\alpha$-DG glycosylation [33]. Furthermore, several missense mutations in ISPD protein have been shown to reduce its enzyme activity, but importantly some residual activities are present. In such cases, abundantly supplied RboP may accelerate the synthesis of CDP-Rbo by mutant ISPD proteins. Although the cellular origin of RboP or its metabolic pathway are unknown, is has been shown that the supplementation of ribitol in drinking water increases cellular CDP-Rbo in wild-type mouse tissues [41]. Thus, ribitol can be also used as a drug. Are such supplementation strategies applicable to fukutin- or FKRP-deficient DGpathies? If missense mutations in these RboP transferases, i.e. fukutin and FKRP, reduce the affinity to their donor substrate CDPribitol, higher concentrations of cellular CDP-ribitol may enhance their enzyme activities. Further studies are necessary to test these ideas.

Important issues to consider are the timing of intervention and the extent of glycosylation recovery. Systemic gene delivery via the tail vein into adolescent skeletal muscle-selective fukutin conditional knock-out mice (Myf5-fukutin cKO) that exhibit early-stage muscular dystrophy dramatically ameliorated the dystrophic phenotype and restored muscle function to the same level as seen in wild-type mice [61]. The data suggest that even after disease manifestation, gene replacement could ameliorate the disease progression. How much restoration of glycosylation is necessary to prevent the progression of the disease? Several cases have been reported in which patients who show only mild muscular dystrophy without 
brain malformations exhibit residual amounts of functionally glycosylated $\alpha$-DG [63]. A study using a transgenic fukutin knock-in mouse carrying a retrotransposal insertion showed that even a small amount of functionally glycosylated $\alpha$-DG is sufficient to maintain skeletal muscle function [64]. These data suggest that effective treatments of DGpathy do not require full recovery of glycosylation.

\section{CONCLUSION}

More than a decade has passed since the establishment of the disease concept now known as DGpathy. During this decade, dedicated efforts have been made to understand the sugar chain structure of $\alpha-D G$ and DGpathy gene functions. Advances in just a few years have resolved these mysteries. In this review, we focused on a novel glycan moiety of $\alpha$-DG, the tandem RboP structure. Fukutin, FKRP, and ISPD are directly involved in the synthesis of the tandem RboP: fukutin and FKRP are RboP transferases, and ISPD is involved in cellular CDP-ribitol synthesis. In Japan, FCMD caused by mutations in fukutin is the dominant form of DGpathy, whereas in the U.S. and Europe, LGMD2I caused by mutations in FKRP is the most prevalent. The number of patients who are diagnosed as carrying ISPD mutations will increase. Muscular dystrophy caused by defects in RboP modification is a subgroup of DGpathy, and many patients fall into this group. Hopefully, therapeutic strategies to correct or enhance RboP modification will be established in the future to overcome these diseases.

\section{ACKNOWLEDGMENTS INCLUDING SOURCES OF SUPPORT}

We thank Drs. Tamao Endo, Yoshiki Yamaguchi, Hiroshi Manya, and Mamoru Mizuno for fruitful comments and discussion. This work was supported by the National Center of Neurology and Psychiatry (NCNP; Intramural Research Grant 29-4 to T.T.), the Japan Agency for Medical Research and Development (AMED; 17930060 to T.T., and 16814830 and 17930144 to M.K.), and the Japan Society for the Promotion of Science (JSPS; 17 H01563 to T.T., and 15H04352 to M.K.).

\section{CONFLICT OF INTEREST}

The authors have no conflict of interest to report.

\section{REFERENCES}

[1] Koenig M, Hoffman EP, Bertelson CJ, Monaco AP, Feener C, Kunkel LM. Complete cloning of the Duchenne muscular dystrophy (DMD) cDNA and preliminary genomic organization of the DMD gene in normal and affected individuals. Cell. 1987;50(3):509-17.

[2] Ervasti JM, Ohlendieck K, Kahl SD, Gaver MG, Campbell KP. Deficiency of a glycoprotein component of the dystrophin complex in dystrophic muscle. Nature. 1990;345 (6273):315-9.

[3] Barresi R, Campbell KP. Dystroglycan: From biosynthesis to pathogenesis of human disease. J Cell Sci. 2006;119(Pt 2):199-207.

[4] Davies KE, Nowak KJ. Molecular mechanisms of muscular dystrophies: Old and new players. Nat Rev Mol Cell Biol. 2006;7(10):762-73.

[5] Hara Y, Balci-Hayta B, Yoshida-Moriguchi T, Kanagawa M, Beltrán-Valero de Bernabé D, Gündeşli H, et al. A dystroglycan mutation associated with limb-girdle muscular dystrophy. N Engl J Med. 2011;364(10):939-46.

[6] Hayashi YK, Ogawa M, Tagawa K, Noguchi S, Ishihara T, Nonaka I, et al. Selective deficiency of alpha-dystroglycan in Fukuyama-type congenital muscular dystrophy. Neurology. 2001;57(1):115-21.

[7] Michele DE, Barresi R, Kanagawa M, Saito F, Cohn RD, Satz JS, et al. Post-translational disruption of dystroglycanligand interactions in congenital muscular dystrophies. Nature. 2002;418(6896):417-22.

[8] Ervasti JM, Campbell KP. A role for the dystrophinglycoprotein complex as a transmembrane linker between laminin and actin. J Cell Biol. 1993;122(4):809-23.

[9] Toda T, Kobayashi K, Takeda S, Sasaki J, Kurahashi H, Kano H, et al. Fukuyama-type congenital muscular dystrophy (FCMD) and alpha-dystroglycanopathy. Congenit Anom (Kyoto). 2003;43(2):97-104.

[10] Michele DE, Campbell KP. Dystrophin-glycoprotein complex: Post-translational processing and dystroglycan function. J Biol Chem. 2003;278(18):15457-60.

[11] Kobayashi K, Nakahori Y, Miyake M, Matsumura K, Kondo-Iida E, Nomura Y, et al. An ancient retrotransposal insertion causes Fukuyama-type congenital muscular dystrophy. Nature. 1998;394(6691):388-92.

[12] Yoshida A, Kobayashi K, Manya H, Taniguchi K, Kano H, Mizuno M, et al. Muscular dystrophy and neuronal migration disorder caused by mutations in a glycosyltransferase, POMGnT1. Dev Cell. 2001;1(5):717-24.

[13] Beltrán-Valero de Bernabé D, Currier S, Steinbrecher A, Celli J, van Beusekom E, van der Zwaag B, et al. Mutations in the O-mannosyltransferase gene POMT1 give rise to the severe neuronal migration disorder Walker-Warburg syndrome. Am J Hum Genet. 2002;71(5):1033-43.

[14] van Reeuwijk J, Janssen M, van den Elzen C, BeltranValero de Bernabé D, Sabatelli P, Merlini L, et al. POMT2 mutations cause alpha-dystroglycan hypoglycosylation and Walker-Warburg syndrome. J Med Genet. 2005;42(12): 907-12.

[15] Brockington M, Blake DJ, Prandini P, Brown SC, Torelli $\mathrm{S}$, Benson MA, et al. Mutations in the fukutin-related protein gene (FKRP) cause a form of congenital muscular dystrophy with secondary laminin alpha2 deficiency and abnormal glycosylation of alpha-dystroglycan. Am J Hum Genet. 2001;69(6):1198-209.

[16] Brockington M, Yuva Y, Prandini P, Brown SC, Torelli S, Benson MA, et al. Mutations in the fukutin-related protein 
gene (FKRP) identify limb girdle muscular dystrophy 2I as a milder allelic variant of congenital muscular dystrophy MDC1C. Hum Mol Genet. 2001;10(25):2851-9.

[17] Longman C, Brockington M, Torelli S, Jimenez-Mallebrera C, Kennedy C, Khalil N, et al. Mutations in the human LARGE gene cause MDC1D, a novel form of congenital muscular dystrophy with severe mental retardation and abnormal glycosylation of alpha-dystroglycan. Hum Mol Genet. 2003;12(21):2853-61.

[18] Godfrey C, Foley AR, Clement E, Muntoni F. Dystroglycanopathies: Coming into focus. Curr Opin Genet Dev. 2011;21(3):278-85.

[19] Chiba A, Matsumura K, Yamada H, Inazu T, Shimizu T, Kusunoki S, et al. Structures of sialylated $O$-linked oligosaccharides of bovine peripheral nerve alpha-dystroglycan. The role of a novel O-mannosyl-type oligosaccharide in the binding of alpha-dystroglycan with laminin. J Biol Chem. 1997;272(4):2156-62.

[20] Yoshida-Moriguchi T, Campbell KP. Matriglycan: A novel polysaccharide that links dystroglycan to the basement membrane. Glycobiology. 2015;25(7):702-13.

[21] Manya H, Chiba A, Yoshida A, Wang X, Chiba Y, Jigami Y, et al. Demonstration of mammalian protein Omannosyltransferase activity: Coexpression of POMT1 and POMT2 required for enzymatic activity. Proc Natl Acad Sci U S A. 2004;101(2):500-5.

[22] Kuwabara N, Manya H, Yamada T, Tateno H, Kanagawa M, Kobayashi K, et al. Carbohydrate-binding domain of the POMGnT1 stem region modulates O-mannosylation sites of $\alpha$-dystroglycan. Proc Natl Acad Sci USA. 2016;113(33): 9280-5.

[23] Stalnaker SH, Hashmi S, Lim JM, Aoki K, Porterfield M, Gutierrez-Sanchez G, et al. Site mapping and characterization of O-glycan structures on alpha-dystroglycan isolated from rabbit skeletal muscle. J Biol Chem. 2010; 285(32):24882-91.

[24] Yoshida-Moriguchi T, Yu L, Stalnaker SH, Davis S, Kunz $\mathrm{S}$, Madson M, et al. O-mannosyl phosphorylation of alphadystroglycan is required for laminin binding. Science. 2010; 327(5961):88-92.

[25] Yoshida-Moriguchi T, Willer T, Anderson ME, Venzke D, Whyte T, Muntoni F, et al. SGK196 is a glycosylationspecific O-mannose kinase required for dystroglycan function. Science. 2013;341(6148):896-9.

[26] Jae LT, Raaben M, Riemersma M, van Beusekom E, Blomen VA, Velds A, et al. Deciphering the glycosylome of dystroglycanopathies using haploid screens for lassa virus entry. Science. 2013;340(6131):479-83.

[27] Manzini MC, Tambunan DE, Hill RS, Yu TW, Maynard TM, Heinzen EL, et al. Exome sequencing and functional validation in zebrafish identify GTDC2 mutations as a cause of Walker-Warburg syndrome. Am J Hum Genet. 2012;91(3):541-7.

[28] Stevens E, Carss KJ, Cirak S, Foley AR, Torelli S, Willer T, et al. Mutations in B3GALNT2 cause congenital muscular dystrophy and hypoglycosylation of $\alpha$-dystroglycan. Am J Hum Genet. 2013;92(3):354-65.

[29] Grewal PK, Holzfeind PJ, Bittner RE, Hewitt JE. Mutant glycosyltransferase and altered glycosylation of alphadystroglycan in the myodystrophy mouse. Nat Genet. 2001;28(2):151-4.

[30] Barresi R, Michele DE, Kanagawa M, Harper HA, Dovico SA, Satz JS, et al. LARGE can functionally bypass alpha-dystroglycan glycosylation defects in distinct congenital muscular dystrophies. Nat Med. 2004;10(7): 696-703.

[31] Kanagawa M, Saito F, Kunz S, Yoshida-Moriguchi T, Barresi R, Kobayashi YM, et al. Molecular recognition by LARGE is essential for expression of functional dystroglycan. Cell. 2004;117(7):953-64.

[32] Inamori K, Yoshida-Moriguchi T, Hara Y, Anderson ME, Yu L, Campbell KP. Dystroglycan function requires xylosyland glucuronyltransferase activities of LARGE. Science. 2012;335(6064):93-6.

[33] Kanagawa M, Kobayashi K, Tajiri M, Manya H, Kuga A, Yamaguchi Y, et al. Identification of a post-translational modification with ribitol-phosphate and its defect in muscular dystrophy. Cell Rep. 2016;14(9):2209-23.

[34] Kanagawa M. Dystroglycan glycosylation and its involvement in muscular dystrophy. Trends Glycosci. Glycotech. 2014;26(149):41-57.

[35] Briggs DC, Yoshida-Moriguchi T, Zheng T, Venzke D, Anderson ME, Strazzulli A, et al. Structural basis of laminin binding to the LARGE glycans on dystroglycan. Nat Chem Biol. 2016;12(10):810-4.

[36] Willer T, Lee H, Lommel M, Yoshida-Moriguchi T, de Bernabe DB, Venzke D, et al. ISPD loss-of-function mutations disrupt dystroglycan O-mannosylation and cause Walker-Warburg syndrome. Nat Genet. 2012;44(5): 575-80.

[37] Roscioli T, Kamsteeg EJ, Buysse K, Maystadt I, van Reeuwijk J, van den Elzen C, et al. Mutations in ISPD cause Walker-Warburg syndrome and defective glycosylation of $\alpha$-dystroglycan. Nat Genet. 2012;44(5):581-5.

[38] Cirak S, Foley AR, Herrmann R, Willer T, Yau S, Stevens E, et al. ISPD gene mutations are a common cause of congenital and limb-girdle muscular dystrophies. Brain. 2013;136(Pt 1):269-81.

[39] Riemersma M, Froese DS, van Tol W, Engelke UF, Kopec $\mathrm{J}$, van Scherpenzeel M, et al. Human ISPD is a cytidyltransferase required for dystroglycan O-mannosylation. Chem Biol. 2015;22(12):1643-52.

[40] Praissman JL, Willer T, Sheikh MO, Toi A, Chitayat $\mathrm{D}$, Lin YY, et al. The functional O-mannose glycan on $\alpha$-dystroglycan contains a phospho-ribitol primed for matriglycan addition. Elife. 2016;5:e14473.

[41] Gerin I, Ury B, Breloy I, Bouchet-Seraphin C, Bolsée J, Halbout M, et al. ISPD produces CDP-ribitol used by FKTN and FKRP to transfer ribitol phosphate onto $\alpha$-dystroglycan. Nat Commun. 2016;7:11534.

[42] Aravind L, Koonin EV. The fukutin protein familypredicted enzymes modifying cell-surface molecules. Curr Biol. 1999;9(22):R836-7.

[43] Kuchta K, Knizewski L, Wyrwicz LS, Rychlewski L, Ginalski K. Comprehensive classification of nucleotidyltransferase fold proteins: Identification of novel families and their representatives in human. Nucleic Acids Res. 2009;37(22):7701-14.

[44] Buysse K, Riemersma M, Powell G, van Reeuwijk J, Chitayat D, Roscioli T, et al. Missense mutations in $\beta$-1,3-N-acetylglucosaminyltransferase 1 (B3GNT1) cause Walker-Warburg syndrome. Hum Mol Genet. 2013; 22(9):1746-54.

[45] Willer T, Inamori K, Venzke D, Harvey C, Morgensen G, Hara Y, et al. The glucuronyltransferase B4GAT1 is required for initiation of LARGE-mediated $\alpha$-dystroglycan functional glycosylation. Elife. 2014;3. Doi: 10.7554/eLife. 03941 
[46] Praissman JL, Live DH, Wang S, Ramiah A, Chinoy $\mathrm{ZS}$, Boons GJ, et al. B4GAT1 is the priming enzyme for the LARGE-dependent functional glycosylation of $\alpha$ dystroglycan. Elife. 2014;3. Doi: 10.7554/eLife.03943

[47] Manya H, Yamaguchi Y, Kanagawa M, Kobayashi K, Tajiri M, Akasaka-Manya $\mathrm{K}$, et al. The muscular dystrophy gene TMEM5 encodes a ribitol $\beta 1,4$-xylosyltransferase required for the functional glycosylation of dystroglycan. J Biol Chem. 2016;291(47):24618-27.

[48] Vuillaumier-Barrot S, Bouchet-Séraphin C, Chelbi M, Devisme L, Quentin S, Gazal S, et al. Identification of mutations in TMEM5 and ISPD as a cause of severe cobblestone lissencephaly. Am J Hum Genet. 2012;91(6):1135-43.

[49] Sheikh MO, Halmo SM, Wells L. Recent advancements in understanding mammalian O-mannosylation. Glycobiology. 2017;27(9):806-19.

[50] Manya H, Endo T. Glycosylation with ribitol-phosphate in mammals: New insights into the O-mannosyl glycan. Biochim Biophys Acta. 2017 in press.

[51] Barone R, Aiello C, Race V, Morava E, Foulquier F, Riemersma M, et al. DPM2-CDG: A muscular dystrophydystroglycanopathy syndrome with severe epilepsy. Ann Neurol. 2012;72(4):550-8.

[52] Lefeber DJ, de Brouwer AP, Morava E, Riemersma M, Schuurs-Hoeijmakers JH, Absmanner B, et al. Autosomal recessive dilated cardiomyopathy due to DOLK mutations results from abnormal dystroglycan O-mannosylation. PLoS Genet. 2011;7(12):e1002427.

[53] Lefeber DJ, Schönberger J, Morava E, Guillard M, Huyben KM, Verrijp K, et al. Deficiency of Dol-P-Man synthase subunit DPM3 bridges the congenital disorders of glycosylation with the dystroglycanopathies. Am J Hum Genet. 2009;85(1):76-86.

[54] Yang AC, Ng BG, Moore SA, Rush J, Waechter CJ, Raymond KM, et al. Congenital disorder of glycosylation due to DPM1 mutations presenting with dystroglycanopathytype congenital muscular dystrophy. Mol Genet Metab. 2013;110(3):345-51.

[55] Carss KJ, Stevens E, Foley AR, Cirak S, Riemersma $\mathrm{M}$, Torelli $\mathrm{S}$, et al. Mutations in GDP-mannose pyrophosphorylase $\mathrm{B}$ cause congenital and limb-girdle muscular dystrophies associated with hypoglycosylation of $\alpha$-dystroglycan. Am J Hum Genet. 2013;93(1):29-41.
[56] Maeda Y, Kinoshita T. Dolichol-phosphate mannose synthase: Structure, function and regulation. Biochim Biophys Acta. 2008;1780(6):861-8.

[57] Fukuyama Y, Osawa M, Suzuki H. Congenital progressive muscular dystrophy of the Fukuyama type - clinical, genetic and pathological considerations. Brain Dev. 1981;3(1):1-29.

[58] Toda T, Kobayashi K, Kondo-Iida E, Sasaki J, Nakamura Y. The Fukuyama congenital muscular dystrophy story. Neuromuscul Disord. 2000;10(3):153-9.

[59] Nakano I, Funahashi M, Takada K, Toda T. Are breaches in the glia limitans the primary cause of the micropolygyria in Fukuyama-type congenital muscular dystrophy (FCMD)? Pathological study of the cerebral cortex of an FCMD fetus. Acta Neuropathol. 1996;91(3):313-21.

[60] Taniguchi-Ikeda M, Kobayashi K, Kanagawa M, Yu CC, Mori K, Oda T, et al. Pathogenic exon-trapping by SVA retrotransposon and rescue in Fukuyama muscular dystrophy. Nature. 2011;478(7367):127-31.

[61] Kanagawa M, Yu CC, Ito C, Fukada S, Hozoji-Inada M, Chiyo T, et al. Impaired viability of muscle precursor cells in muscular dystrophy with glycosylation defects and amelioration of its severe phenotype by limited gene expression. Hum Mol Genet. 2013;22(15):3003-15.

[62] Xu L, Lu PJ, Wang CH, Keramaris E, Qiao C, Xiao B, et al. Adeno-associated virus 9 mediated FKRP gene therapy restores functional glycosylation of $\alpha$-dystroglycan and improves muscle functions. Mol Ther. 2013;21(10): 1832-40.

[63] Murakami T, Hayashi YK, Noguchi S, Ogawa M, Nonaka I, Tanabe Y, et al. Fukutin gene mutations cause dilated cardiomyopathy with minimal muscle weakness. Ann Neurol. 2006;60(5):597-602.

[64] Kanagawa M, Nishimoto A, Chiyonobu T, Takeda S, Miyagoe-Suzuki Y, Wang F, et al. Residual lamininbinding activity and enhanced dystroglycan glycosylation by LARGE in novel model mice to dystroglycanopathy. Hum Mol Genet. 2009;18(4):621-31.

[65] Praissman JL, Wells L. Mammalian O-mannosylation pathway: Glycan structures, enzymes, and protein substrates. Biochemistry. 2014;53(19):3066-78. 